\section{Algorithmic Self-Assembly of DNA: Theoretical Motivations and 2D Assembly Experiments}

\author{
http://www.adeninepress.com/jbsd
}

\begin{abstract}
Biology makes things far smaller and more complex than anything produced by human engineering. The biotechnology revolution has for the first time given us the tools necessary to consider engineering on the molecular level. Research in DNA computation, launched by Len Adleman, has opened the door for experimental study of programmable biochemical reactions. Here we focus on a single biochemical mechanism, the self-assembly of DNA structures, that is theoretically sufficient for Turing-universal computation. The theory combines Hao Wang's purely mathematical Tiling Problem with the branched DNA constructions of Ned Seeman. In the context of mathematical logic, Wang showed how jigsaw-shaped tiles can be designed to simulate the operation of any Turing Machine. For a biochemical implementation, we will need molecular Wang tiles. DNA molecular structures and intermolecular interactions are particularly amenable to design and are sufficient for the creation of complex molecular objects. The structure of individual molecules can be designed by maximizing desired and minimizing undesired Watson-Crick complementarity. Intermolecular interactions are programmed by the design of sticky ends that determine which molecules associate, and how. The theory has been demonstrated experimentally using a system of synthetic DNA double-crossover molecules that self-assemble into two-dimensional crystals that have been visualized by atomic force microscopy. This experimental system provides an excellent platform for exploring the relationship between computation and molecular self-assembly, and thus represents a first step toward the ability to program molecular reactions and molecular structures.
\end{abstract}

\section{Introduction}

An E. Coli bacterium is approximately $1 \mu \mathrm{m}^{3}$ - roughly the same size as a single transistor in a modern computer chip. Instead of storing a single bit of information, E. Coli stores more than a megabyte of genetic information in its DNA. This is just one measure by which biological technology vastly outperforms current industrial technology.

Len Adleman realized that the density of information storage in DNA, combined with the parallelism of chemical reactions, potentially could be used to perform huge mathematical computations - and thus he invented the field of DNA-based computers (Adleman 1994, Lipton and Baum 1996; Landweber and Baum 1998; Rubin and Wood 1999). The essential statistics are that $1 \mathrm{ml}$ of solution can contain $10^{21}$ bits of information encoded as DNA, and that chemical reactions (such as the activity of restriction enzymes, ligases, polymerases, or simple hybridization itself) can operate on this data in parallel. Questions of what biochemical operations are best for molecular computation, how best to use them, whether they can be performed with sufficiently low errors, and what specific applications are likely to be of practical interest, are all still open.

It is particularly interesting to consider the variety of mechanisms used to control intracellular processes, in order to understand the structure of algorithms that these

\author{
Erik Winfree ${ }^{\dagger *}$ \\ Computation and Neural Systems, \\ California Institute of Technology, \\ Pasadena, CA 91125 \\ $\dagger$ Current address: \\ Department of Molecular Biology, \\ Princeton University, \\ Princeton, NJ 08544
}

*Phone: 626-395-6246; Fax: 626-792-4257;

E-mail: winfree@caltech.edu 
types of processes can implement. A general theory of molecular computation ought to suggest how to design synthetic biochemical systems to perform any chosen algorithm. Research on ribosomal translation (Bennett 1982; Kurtz et al. 1997), signal transduction (Hjelmfelt and Ross 1995; Magnasco 1997), genetic regulatory circuits (Weiss et al. to appear), and self-assembly (Winfree 1996) has already begun.

In this paper we explain the central idea of computation by selfassembly of DNA as proposed in Winfree (1996), and present additional characterization of the experimental system of Winfree et al. (1998a).

\subsection{Computation by Tiling}

The Tiling Problem was introduced by Hao Wang to study a question in mathematical logic (Wang 1961). The problem is a simple challenge: given a finite set of geometrical tiles (e.g. polygons), determine whether they can be arranged (using each tile as many times as necessary) to cover the entire plane without gaps. The remarkable conclusion, due to Wang's student Robert Berger, is that there does not exist any algorithm that can give the correct answer in every case: the Tiling Problem is undecidable (Berger 1966). This result relies on constructions wherein tiling patterns simulate single-tape Turing Machines (Buchi 1962; Wang 1963; Robinson 1971; Wang 1975), and this is where our interest lies, because these constructions highlight a simple but deep connection between the process of fitting geometrical objects together and computation itself. Tilings and patterns in general are surveyed in a beautiful book by Grünbaum and Shepard (1987).

Computation by tiling is best introduced by example. We begin with the tiles in Figure 1. It is easy to see that these two tiles can tile the plane in a unique way, forming a striped, periodic lattice. The shapes of the left and right sides of the tiles determines how the tiles can be placed next to each other. The tiles here can be thought of as having four binding domains: the upper left, lower left, upper right, and lower right sides. To enforce a different pattern, one only needs to change the shapes of these four domains. This is done in Figure 2, where we now have four tiles forming a striped, periodic lattice with twice the period. Neither of these examples involves any significant computation, but they demonstrate that local shape compatibility can determine the global arrangement of units.

Our first computational example is shown in Figure 3. These tiles implement a binary counter. The goal is simply to count 1 , $2,3,4,5, \ldots$, but in base 2 , where it would be $1,10,11,100,101$, ..., with subsequent numbers written above the previous ones. Shapes on the tiles represent information used in the computation: the top and bottom sides encode the value of bits in the counter, while the left and right sides are use to carry the rollover bit (more familiar in base 10, where 9 rolls over to 0 and increments the digit to its left). The seed tile $\mathrm{S}$ triggers the computation, and the two boundary tiles (bottom and right) provide initial conditions. The bottom boundary tile produces a series of rounded tops, encoding the initial counter bits ...000. The bound- ary tiles on the right produce a series of "commands" to increment the rightmost bit in every row. The four rule tiles can then be seen to effect the following logic: "if there is no rollover from the bit on the right, this bit stays the same; but if there is a rollover from the bit on the right, 0 becomes 1 , and 1 rolls over to 0 ."

It is important to note that there are two new ingredients in this example. First, to ensure that the correct pattern results, the three input tiles are assembled before the rule tiles are used. (This restriction can be relaxed somewhat, but it is convenient for explanation.) Second, to avoid getting stuck with an incorrect partial tiling, one must add rule tiles only when both the tiles below and to their right are already present. This condition also prevents the formation of tilings in the absence of the input assembly, such as an infinite periodic lattice containing only the all-0 tile. To use a biochemist's terminology, we require that binding be a highly cooperative event, and the reaction must take place at a temperature above the melting temperature of the single binding domain and below the melting temperature of a pair of cooperative binding domains.

In the general case, this kind of tiling process can perform any kind of information processing task - that is to say, it is Turinguniversal. A computer program can be automatically translated into a set of tiles that perform exactly the same mathematical function. Again, all that is necessary is redesigning the four binding domains on the sides of the rectangular tiles; rectangular tiles, called Wang tiles are therefore of particular importance. The general framework is illustrated in Figure 4. Input to the system is provided in the form of a preassembled arrangement of tiles whose shape (in the binding domains) encodes the mathematical value of the input. The computation proceeds as before, resulting in an upper layer that encodes the output in the same form as the input. As in the binary counter example, tile sets are typically designed such that a unique tile can fit into each location, and thus a unique tiling is deterministically produced.

It is also possible that a set of tiles do not deterministically produce a unique tiling. In this case, there are many possible valid tilings, any or all of which may be produced. When tiles are implemented by real molecules, one would expect a set of nondeterministic tiles to generate a combinatorial library. This is exactly what is necessary to perform a massively parallel computation. As sketched in Figure 5, a non-deterministic set of tiles could produce a combinatorial library of input assemblies (the first layer of the tiling), and then a deterministic set of rule tiles could evaluate each input assembly to determine whether it represents the desired answer.

\subsection{Design of DNA Crystal}

Our goal is to design synthetic molecular units corresponding to Wang tiles, such that they will self-assemble into a crystal that obeys the matching rules. Specific interactions between binding domains can be implemented by sequence-specific hybridization of single-strand DNA to form double helical DNA. Wang tiles require four binding domains, and thus four available sticky 


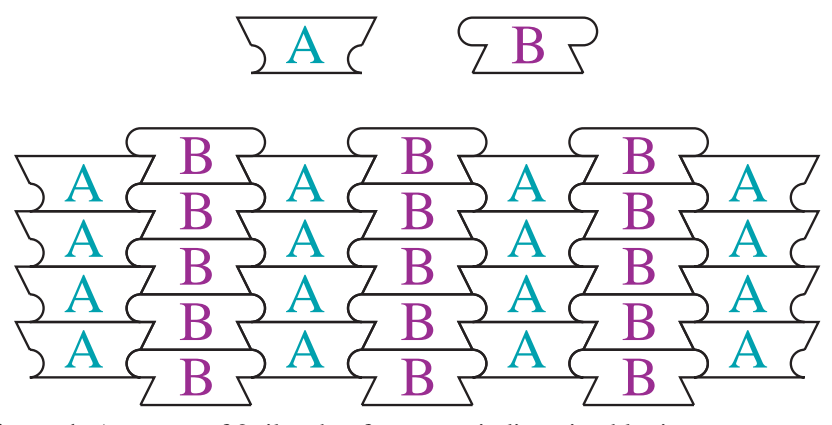

Figure 1: A system of 2 tiles that form a periodic striped lattice.
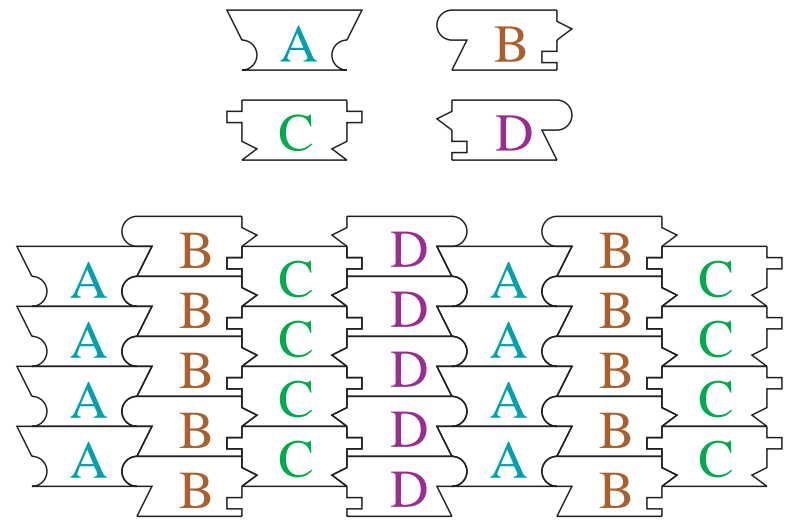

Figure 2: A system of 4 tiles that form a periodic striped lattice.

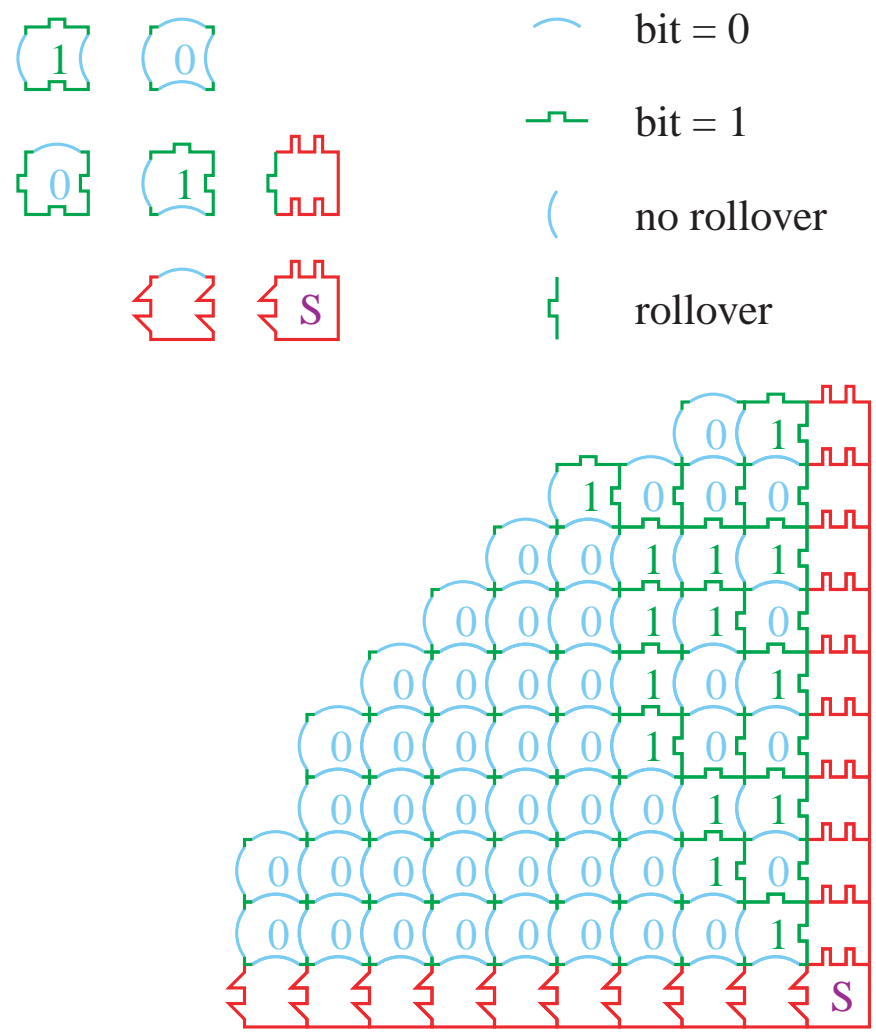

Figure 3: A system of 3 input tiles and 4 rule tiles that form an aperiodic tiling. The rows in the tiling are the consecutive integers, represented in binary. ends; this necessitates the use of DNA double-crossover (DX) molecules (Fu and Seeman 1993) or other branched constructs.

This study uses molecules whose design was previously reported (Winfree et a. 1998a). As an initial demonstration of molecu-

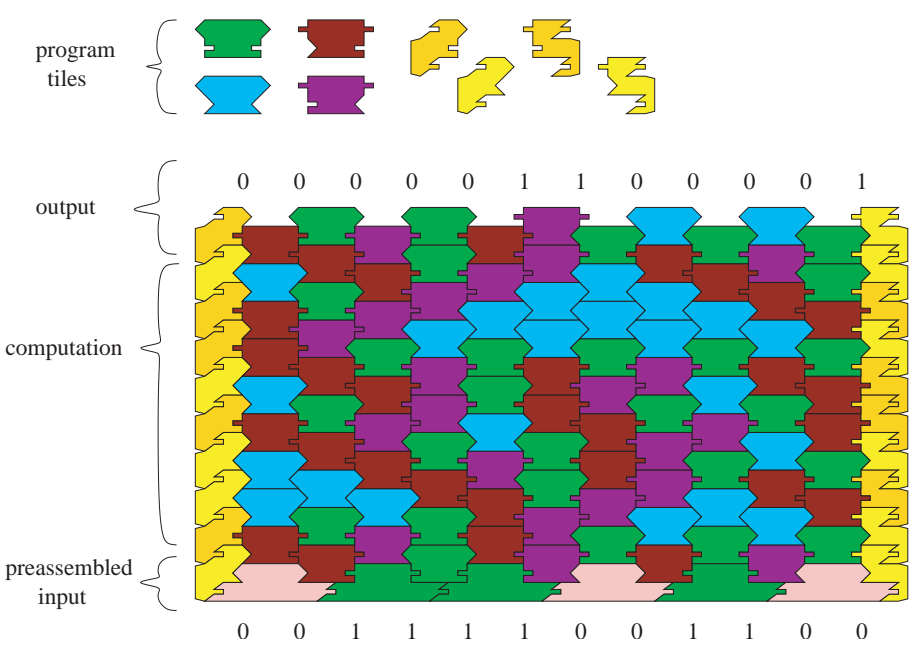

Figure 4: The framework for universal computation by tiling. An input arrangement is presented at the bottom. The sequence of exposed shapes encodes the input information. The program, a set of rule tiles (top), determines the continuation of the tiling pattern. The output is encoded in the final, uppermost layer of the tiling.

lar Wang tiles, we chose the simplest non-trivial set of tiles: the two tiles, $\mathbf{A}$ and $\mathbf{B}$, from Figure 1. Translated into molecular terms, as shown in Figure 6, we obtain a DX system (using the DAO variety of DX (Fu and Seeman 1993)) that self-assembles in solution into two-dimensional crystals with a well-defined subunit structure, as reported in Winfree et al. (1998a).

\section{Materials and Methods}

\subsection{DNA Sequences and Synthesis}

All oligonucleotides were synthesized by standard methods, PAGE purified, and quantitated by UV absorption at $260 \mathrm{~nm}$ in $\mathrm{H}_{2} \mathrm{O}$. The exact sequences are given in Winfree et al. (1998a).

\subsection{Annealing of Oligonucleotides}

The strands of each DX unit were mixed stoichiometrically and dissolved to concentrations of 0.2 to $2 \mu \mathrm{M}$ in TAE/ $\mathrm{Mg}^{++}$buffer (40 mM Tris $\cdot \mathrm{HCl}$ (pH 8.0), $1 \mathrm{mM}$ EDTA, $3 \mathrm{mM} \mathrm{Na}{ }^{+}, 12.5 \mathrm{mM}$ $\mathrm{Mg}^{++}$). The solutions were annealed from $90^{\circ} \mathrm{C}$ to room temperature over the course of several hours in a Perkin-Elmer PCR machine (to prevent concentration by evaporation). To produce lattices, equal amounts of each DX were mixed and annealed from $50^{\circ} \mathrm{C}$ to $20^{\circ} \mathrm{C}$ over the course of up to 36 hours. In some cases (Figure 11abc and Figure 10def) all strands were mixed together from the very beginning.

\subsection{Gel Electrophoresis Studies}

For gel-based studies, T4 polynucleotide kinase (Amersham) was used to phosphorylate strands with ${ }^{32} \mathrm{P}$; these strands were then PAGE purified and mixed with an excess of unlabeled strands. Nondenaturing 5\% PAGE (19:1 acrylamide:bis-acrylamide) in $\mathrm{TAE} / \mathrm{Mg}^{++}$was performed at $4^{\circ} \mathrm{C}$. For denaturing experiments, after annealing in T4 DNA ligase buffer (Amersham) $(66 \mathrm{mM}$ Tris $\cdot \mathrm{HCl}$ 


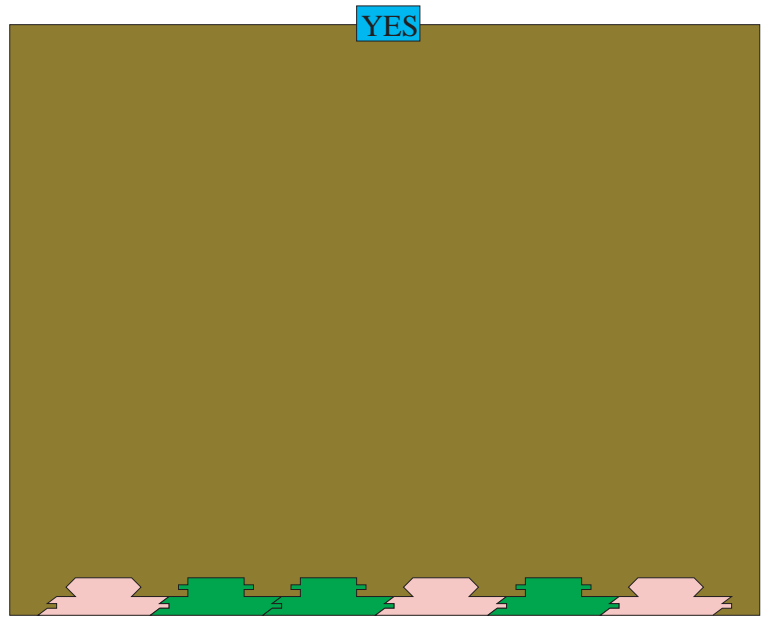

Figure 5: Using self-assembly to solve NP-complete problems. A combinatorial library of inputs is generated. Computation by tiling proceeds, either including

(pH 7.6), $6.6 \mathrm{mM} \mathrm{MgCl}_{2}, 10 \mathrm{mM}$ DTT, $66 \mu \mathrm{M}$ ATP), $1 \mu \mathrm{L}=10$ units T4 DNA ligase (Amersham) was added to $10 \mu \mathrm{L}$ DNA solution and incubated for up to 24 hours at $16^{\circ} \mathrm{C}$. The solution was added to an excess of denaturing dye buffer $(0.1 \%$ xylene cyanol FF tracking dye in $90 \%$ formamide with $1 \mathrm{mM} \mathrm{EDTA}, 10 \mathrm{mM} \mathrm{NaOH}$ ) and heated to $90^{\circ} \mathrm{C}$ for at least 5 minutes prior to loading. Denaturing gels contained $4 \%$ acrylamide (90:1 acrylamide:bisacrylamide) and $8.3 \mathrm{M}$ urea in TBE ( $89 \mathrm{mM}$ Tris $\cdot \mathrm{HCl}$ (pH 8.0), $89 \mathrm{mM}$ boric acid, $2 \mathrm{mM}$ EDTA). Gels were analyzed by phosphorimager.

\subsection{Preparation of AFM Sample}

For all AFM studies, stock solutions were annealed at $.2 \mu \mathrm{M}$ each DNA strand. 2 to $10 \mu \mathrm{L}$ were spotted on freshly cleaved mica (Ted

\section{A}

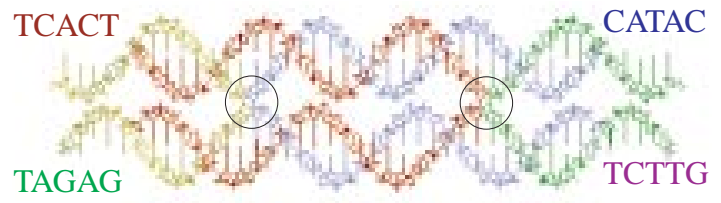

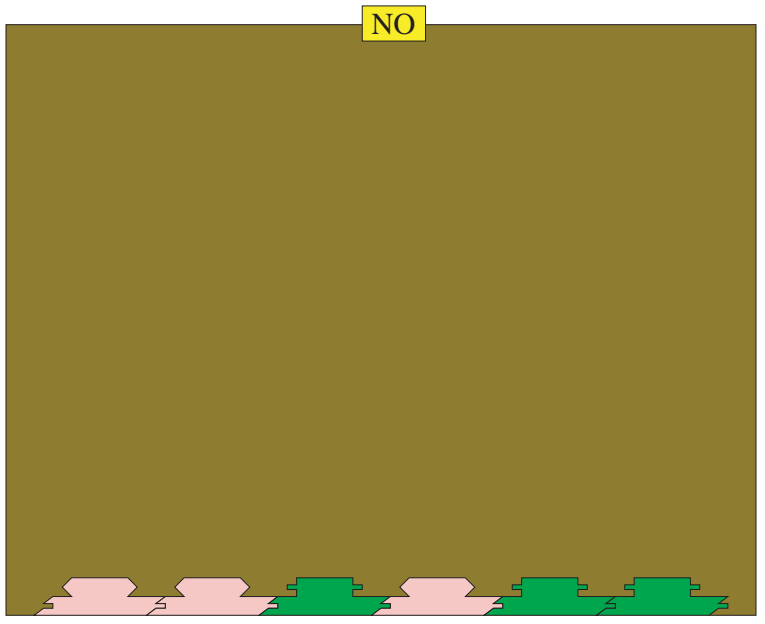

the "YES" tile or the "NO" tile for each input. The presence of a "YES" tile must then be amplified by some means; PCR can be used if the tiles are made of DNA.

Pella, Inc) and left to adsorb to the surface for 2 minutes. In some cases Figure 10def) the solution was diluted 10-fold in $\mathrm{H}_{2} 0$ prior to spotting on mica, because the stock solution resulted in too dense coverage of the surface. To remove buffer salts, 5 to 10 drops of doubly-distilled or nanopure $\mathrm{H}_{2} \mathrm{O}$ were placed on the mica, the drop was shaken off and the sample was dried with compressed air. Imaging was performed under isopropanol in a fluid cell on a NanoScope II using the D or E scanner and commercial $200 \mu \mathrm{m}$ cantilevers with $\mathrm{Si}_{3} \mathrm{~N}_{4}$ tips (Digital Instruments). The feedback setpoint was adjusted frequently to minimize contact force to approximately 1 to $5 \mathrm{nN}$. Images were processed with a first- or third-order "flatten filter," which independently subtracts a first- or third-order polynomial fit from each scanline to remove tip artifacts; however, this technique introduces false "shadows" into the images shown here.

\section{B}

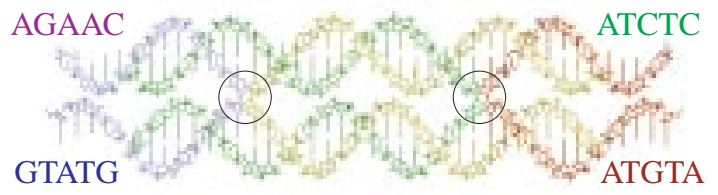

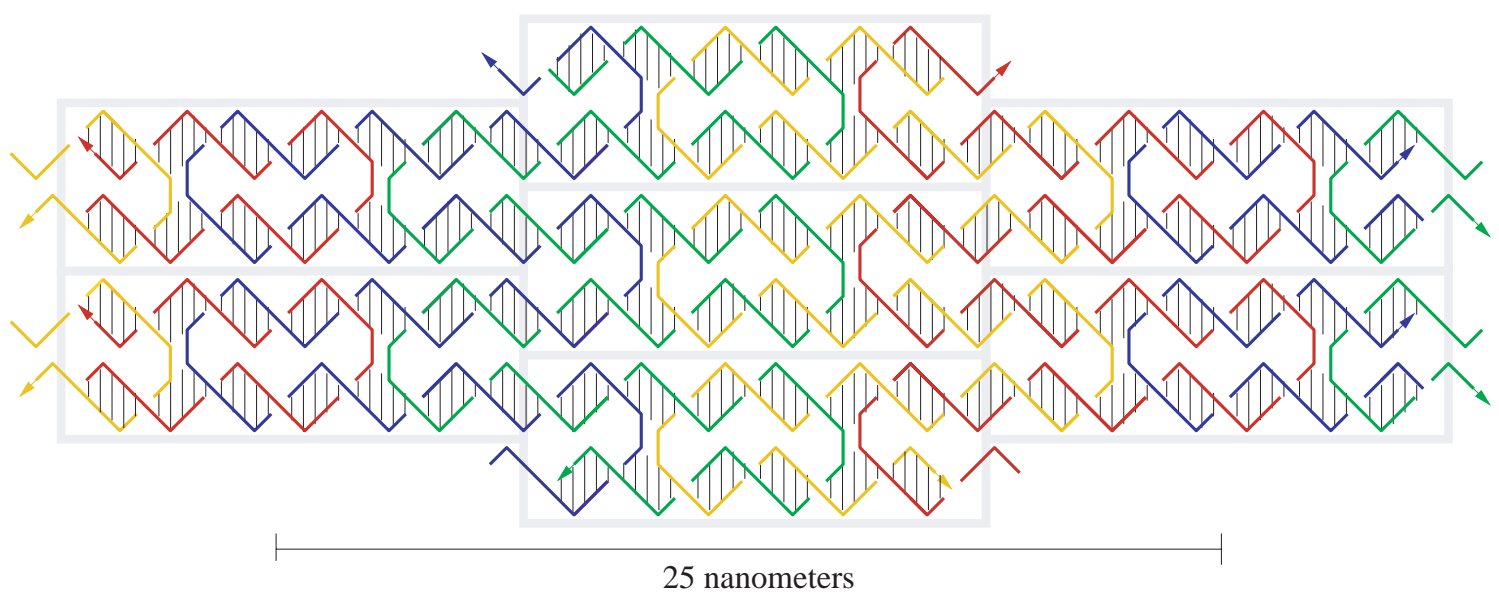

Figure 6: Design of DX molecular structure and arrangement into 2-D lattices. (top) Model structures for DAO units $\mathbf{A}$ and $\mathbf{B}$. Each component oligonucleotide is shown in a unique color. The crossover points are circled. (below) The lattice topologies produced by the DAO. Each DX unit is highlighted by a grey rectangle. A unique color is chosen for each strand type that would be formed after covalent ligation of units. Arrowheads indicate the $3^{\prime}$ ends of strands. 
A $5 \%$ Non-denaturing PAGE $\quad$ B

$\begin{array}{lllllllllll}1 & 2 & 3 & 4 & 5 & 6 & 7 & 8 & 9 & 10 & 11\end{array}$

$\begin{array}{lllllllllll}1 & 2 & 3 & 4 & 5 & 6 & 7 & 8 & 9 & 10 & 11\end{array}$
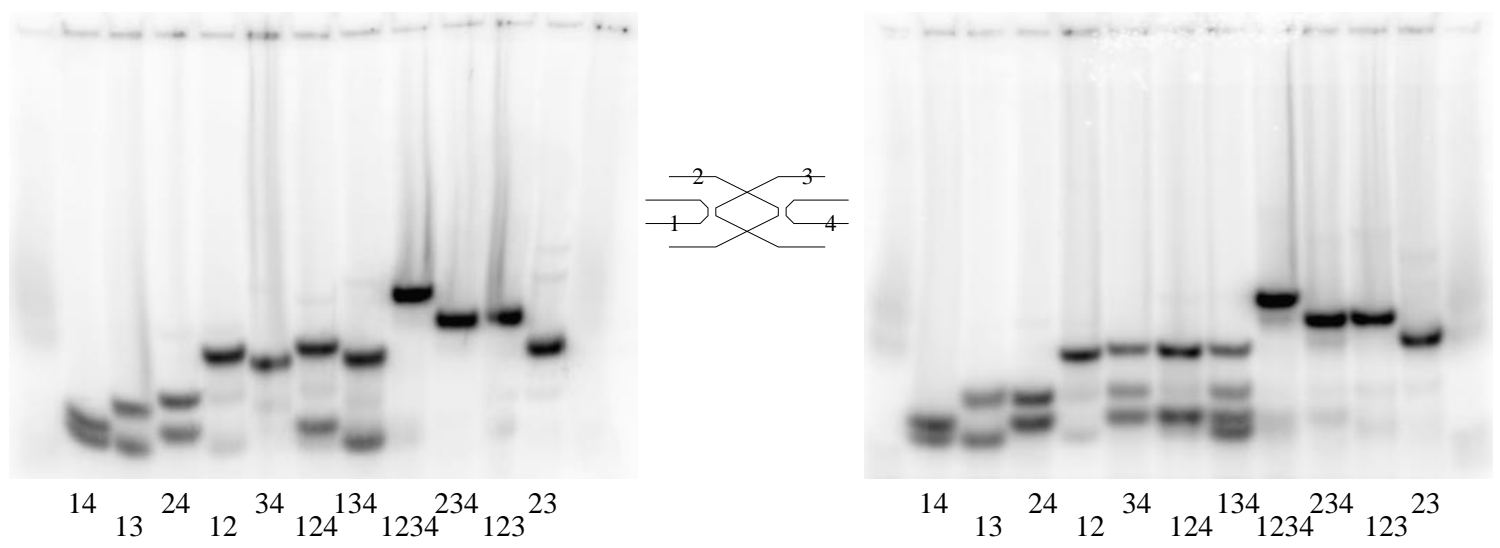

Figure 7: Formation gels for the $\mathbf{A}$ and $\mathbf{B}$ double-crossover molecules, run at $1{ }^{\circ} \mathrm{C}$ by $5 \%$ non-denaturing PAGE. Every strand is radiolabelled so that 3 Results and Discussion

\subsection{Results of Characterization by Gel Electrophoresis}

A prerequisite for lattice self-assembly is the formation of the DX units from their component strands. A thorough investigation of this issue was done for the original studies of DX (Fu and Seeman 1993); that the new designs also behave well constitutes further validation of the antiparallel DX motif. Because the sticky ends of $\mathbf{A}$ units have affinity only for sticky ends of $\mathbf{B}$ units, and not for themselves, neither $\mathbf{A}$ nor $\mathbf{B}$ alone in solution can assemble into a lattice. Thus the formation of isolated DX units can be monitored easily by non-denaturing gel electrophoresis, as described previously (Fu and Seeman 1993), and greater than $95 \%$ of the material is seen in the expected band for A and greater than $85 \%$ for B (Figure 7). Additionally, complexes are formed only by strands which were designed to interact. However, strand 3 of $\mathbf{B}$ does not bind fully to strand 4, unless strand 2 is also present. This may be due to a potential hairpin structure near the $5^{\prime}$ end of strand 3; when strand 2 binds

quantitation is possible. Only the strands indicated below each lane were included in the sample; lane 8 is the complete DX molecule.

to strand 3, we postulate that this hairpin is undone. Note also that dimer and multimer species are not found, and in particular note that the individual $\mathbf{A}$ or $\mathbf{B}$ monomers do not assemble into extended structures, such as polymers or lattices.

Solutions containing $\mathbf{A}$ units and $\mathbf{B}$ units can be mixed and annealed to form AB lattices. Lane 4 of Figure 8 (left) shows that the self-assembled structure is too large to migrate through the gel, although a fraction of the material is coming out of the well in a smear, presumably due to slow dissociation of the lattice. Enzymatic ligation of these lattices with T4 DNA ligase produces immobile material (circled), while the $\mathbf{A}$ and $\mathbf{B}$ units alone are not substrates for ligation (Figure 8 (left)). The nicks in the lattice, where strands from adjacent DX units abut, are all on the upper or lower surface of the lattice, where they are accessible to the enzyme. The ligated lattice should contain long covalent DNA strands (...-A1-B2-A1-B2-..., ...-A2-B1-A2-B1-.., ...-A3-B4-A3B4-..., and ...-A4-B3-A4-B3-...), which serve as reporters of successful lattice formation. Shorter strands report either the presence of small aggregates or the expected occasional failure to

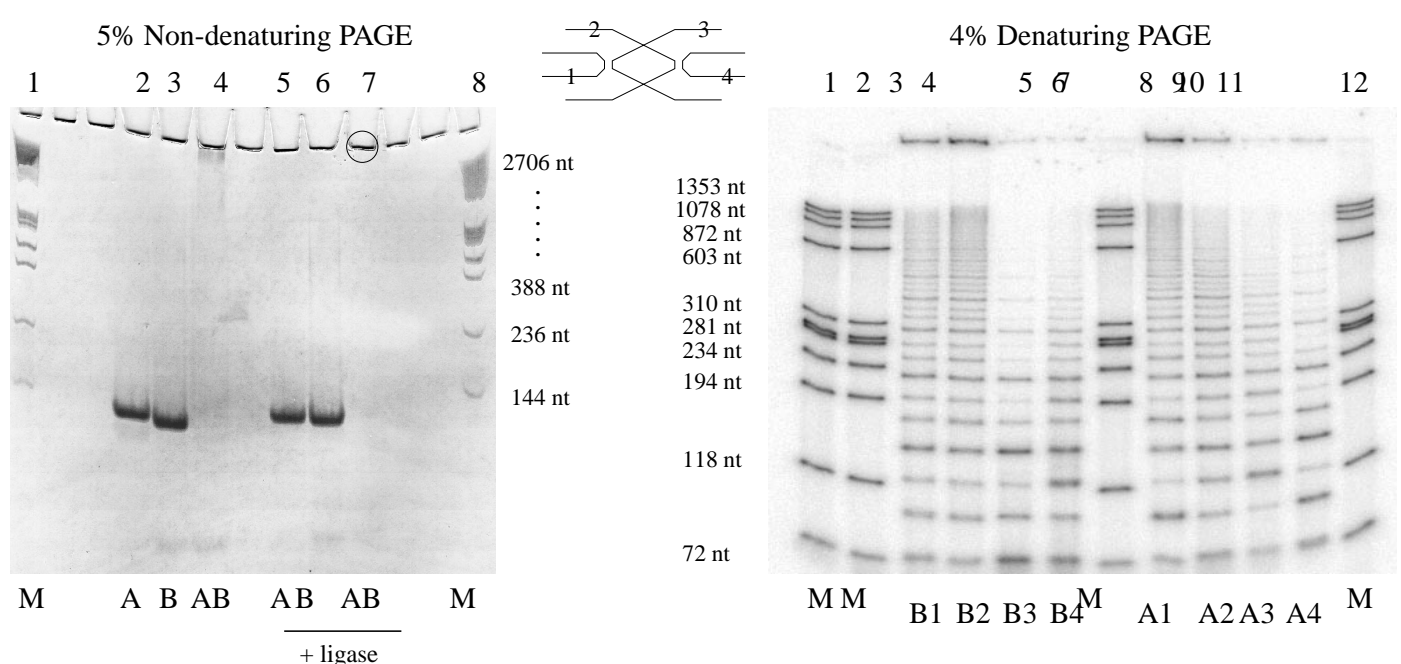

Figure 8: (left) Non-denaturing gel, StainsAll stain. Lanes 2-4 show A alone, $\mathbf{B}$ alone, and $\mathbf{A B}$ together. Lanes 5-7 show $\mathbf{A}$ alone ligated, $\mathbf{B}$ alone ligated and AB together ligated. All the material in lane 7 is in a sharp band directly around the well (circled), as can be seen clearly in color, but not in the rendi-

tion here. There is no material in the wells in lanes 2, 3, 5, 6; the bands are shadows. (right) Denaturing gel, with differential radiolabelling. Every lane contains either marker or ligated AB; in each lane the radiolabelled oligonucleotide is indicated below. 
$5 \%$ Non-denaturing PAGE

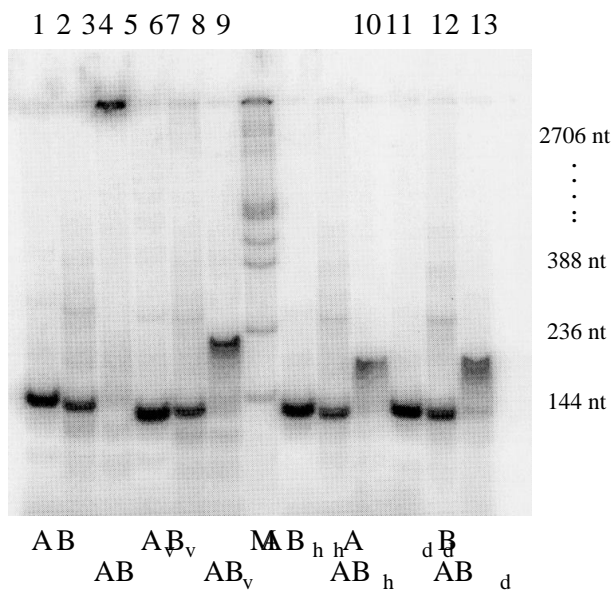

Figure 9: (left) $5 \%$ Non-denaturing gel at $4^{\circ} \mathrm{C}$, with radiolabelling of $\mathbf{A} 3$ and $\mathbf{B} 3$. (right) Diagram of the truncated DX units, and intended chain structures. Units

ligate within the lattice. Band intensities are compatible with a greater than $75 \%$ ligation probability at each nick. Reporter strands extend for more than 30 repeats when visualized by denaturing polyacrylamide gel electrophoresis (Figure 8, right); however, ligation is less extensive for the reporter strands containing $\mathbf{B} 3$ and $\mathbf{B} 4$ and the postulated hairpin. These results suggest that the lattice is a good substrate for T4 DNA ligase, and that the lattices can form with more than $30 \times 30$ units. However, unintended associations or side reactions could lead to similar distributions of strand lengths after ligation. Direct physical observation is necessary to confirm lattice assembly.

As a control to test the 4-connected nature of the putative lattice product, three modified versions of $\mathbf{A}$ and $\mathbf{B}$ were made, each with two sticky ends truncated to blunt ends, as shown in Figure 9. $\mathbf{A}_{v}$ and $\mathbf{B}_{v}$ were designed to make vertical one-dimensional chains of DX units; $\mathbf{A}_{h}$ and $\mathbf{B}_{h}$ were designed to make horizontal one-dimensional chains of DX units; and $\mathbf{A}_{d}$ and $\mathbf{B}_{d}$ were designed to make diagonal one-dimensional chains of DX units. However, quite unlike the $\mathbf{A B}$ product stuck in the well, all three truncated systems produced what appear to be dimers on the non-denaturing gel. No ligase was used. Apparently, the chain structures, if formed, are falling apart into dimers in the gel.

\subsection{Results of AFM Imaging}

We have used atomic force microscopy (Binnig et al. 1986) to demonstrate unequivocally the formation of 2-D lattices (Winfree et al. 1998a). A and B units are annealed separately, then combined and annealed together to form $\mathbf{A B}$ lattices. The resulting solution is deposited for adsorption on an atomically flat mica surface, and then imaged under isopropanol by contact mode AFM (Hansma et al. 1992). The solution is not treated with DNA ligase, and thus the lattices are held together only by noncovalent interactions (e.g. hydrogen bonds and base stacking). This protocol ensures that the solution contains no protein contaminants and demonstrates that ligase activity is not necessary for the self-assembly process. Negative controls of buffer

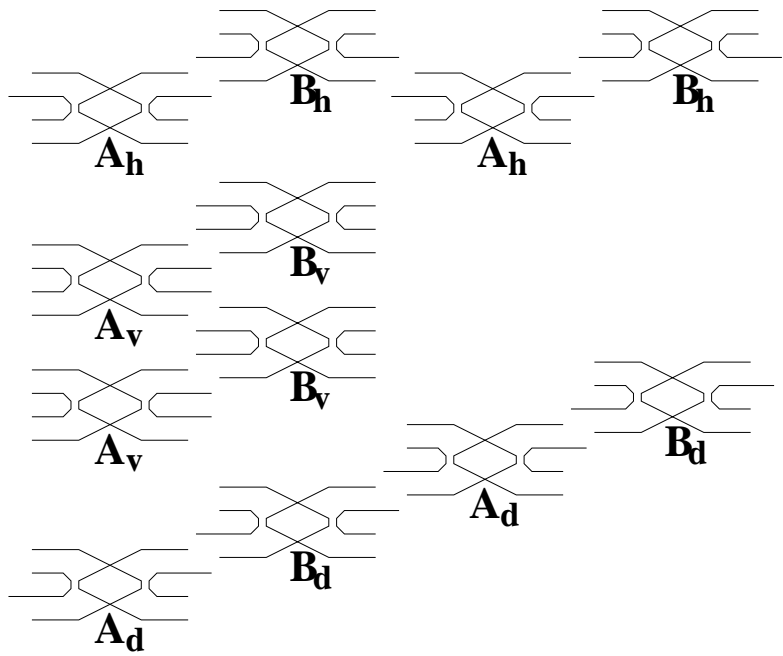

are not ligated.

alone and of $\mathbf{A}$ or $\mathbf{B}$ alone show no objects larger than $20 \mathrm{~nm}$ (Figure 10abc). In separate experiments, $\mathbf{A}$ and $\mathbf{B}$ units were modified by the removal of two sticky ends from each unit; when the modified $\mathbf{A}$ and $\mathbf{B}$ units were annealed together, we observed only linear and branched structures with apparent widths typically less than $10 \mathrm{~nm}$ (Figure 10def), providing additional negative controls. However, the unmodified AB samples contain 2-D sheets many microns long, often more than $200 \mathrm{~nm}$ wide (Figure 11a). The apparent height of the sheets is $1.4 \pm .5$ $\mathrm{nm}$, suggesting a monolayer of DNA. The sheets often seem ripped and appear to have a grain, in that rips have a preferred direction consistent with the design (Figure 6c). In the AB lattice, a vertical rip requires breaking six sticky-end bonds per 12 $\mathrm{nm}$ torn, whereas a horizontal rip requires breaking only one sticky-end bond per $13 \mathrm{~nm}$ torn. A possible vertical column, perpendicular to the rips, is indicated in Figure 11a (arrows). Although in this image the columns are barely perceptible, Fourier analysis shows a peak at $13 \pm 1 \mathrm{~nm}$, suggesting that observed columns are 1 DX wide. Periodic topographic features would not be expected in AFM images in the ideal AB lattice at
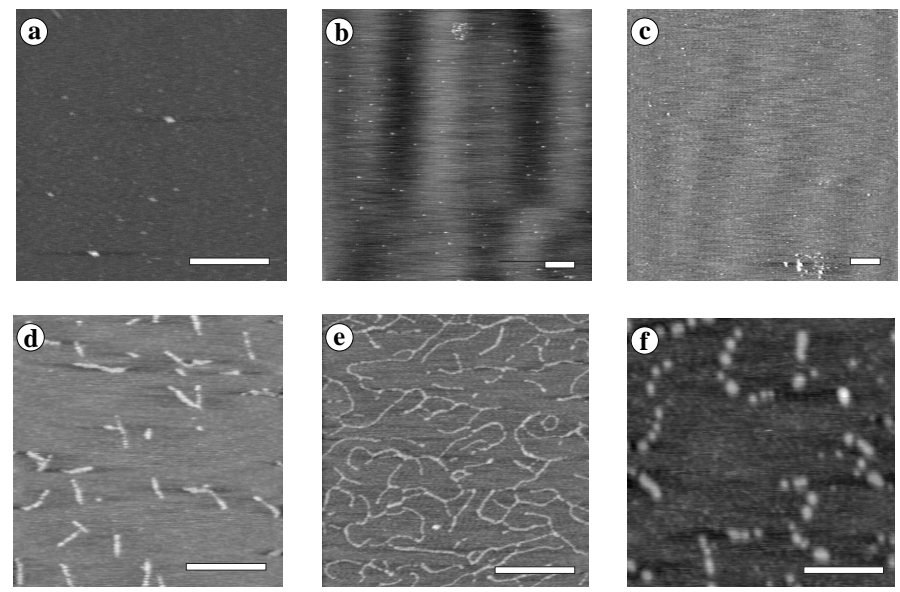

Figure 10: AFM images of buffer (a), $\mathbf{A}$ and $\mathbf{B}$ controls (b and c respectively), and sticky-end-truncation controls $\mathbf{A} \mathbf{B}_{v}(\mathrm{~d}), \mathbf{A} \mathbf{B}_{h}(\mathrm{e})$, and $\mathbf{A} \mathbf{B}_{d}$ (f). All scale bars are $300 \mathrm{~nm}$; images show $1 \times 1 \mu \mathrm{m}$, or $3 \times 3 \mu \mathrm{m}$. The grayscale indicates height above the mica surface; apparent height of features is less than $5 \mathrm{~nm}$. 

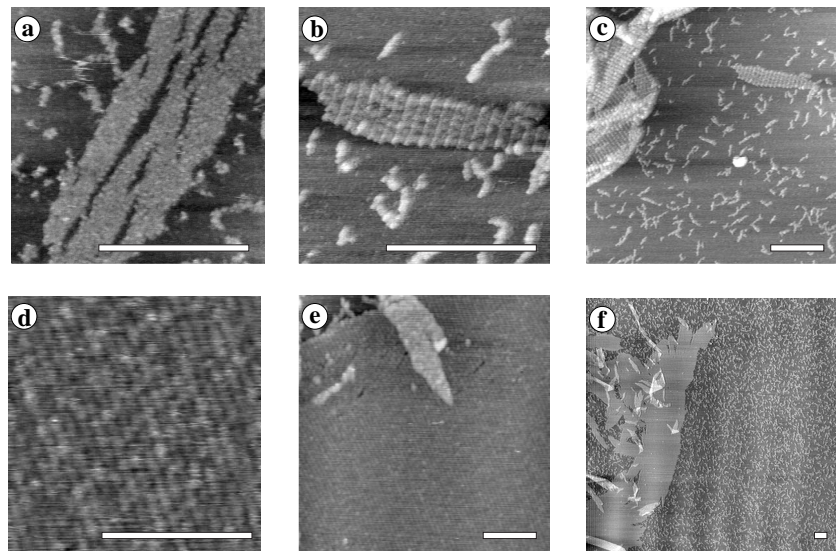

Figure 11: AFM images of unmodified AB lattice (a). A possible vertical column is indicated by the arrows. Fourier analysis shows $13 \pm 1 \mathrm{~nm}$ periodicity; each DAO is $12.6 \mathrm{~nm}$ wide. (b) and (c) show $\mathbf{A B}$ lattice (two views of the same sample). Stripes have $25 \pm 2 \mathrm{~nm}$ periodicity; the expected value is $25.2 \mathrm{~nm}$. (def) show a large single-domain crystal of $\mathrm{AB}$ lattice at three levels of detail (all the same sample). The largest domain is roughly $2 \times 8 \mu \mathrm{m}$, and contains roughly 500,000 DX units. All scale bars are $300 \mathrm{~nm}$; images show $500 \times 500 \mathrm{~nm}, 1.5$ $\times 1.5 \mu \mathrm{m}$, or $10 \times 10 \mu \mathrm{m}$. The grayscale indicates height above the mica surface; apparent lattice height is between 1 and $2 \mathrm{~nm}$.

this resolution; however a vertically stretched lattice may have gaps between the DX units that could produce the periodic features seen here. Because crystals are found in AFM samples taken from both the top and the bottom of the solution, we believe that crystals form in solution and are not due to interaction with a surface. Crystals are not seen if the annealing is aborted prematurely at a high temperature (data not shown).

\subsection{Control of Surface Topography}

The self-assembling $\mathbf{A B}$ lattice can serve as scaffolding for other molecular structures. As reported in Winfree et al. (1998a), we have decorated $\mathbf{B}$ with two DNA hairpin sequences inserted into its component strands, which we call $\hat{\mathbf{B}}$. So decorated, the vertical columns of the lattice become strikingly apparent as stripes in AFM images (Figure 11bc), further confirming the proper self-assembly of the 2-D lattice. The spacing of the decorated columns is $25 \pm 2 \mathrm{~nm}$ for the $\mathbf{A B}$ lattice, indicating that every other column is decorated, in accord with the design. Slow annealing at $20^{\circ} \mathrm{C}$ and gentle handling of the sample during deposition and washing has produced single crystals measuring up to $2 \times 8 \mu \mathrm{m}$ (Figure 11def). Close examination shows that the stripes are continuous across the crystal, and thus it appears to be a single domain containing over 500,000 DX units.

We have also tested DAO systems incorporating only one of the two hairpins in $\hat{\mathbf{B}}$ and DAO systems in which the 3 -arm junctions are relocated by two nucleotides toward the center of the molecule. All systems produced results similar to those shown in Figure 11 when imaged by AFM (data not shown). The lattice assembly appears to be robust to variations in the local DX structure and is not sensitive to small variations in the annealing protocol. (Also see Winfree et al. 1998a) for similar results obtained in another laboratory using different buffers, annealing conditions, and AFM instruments.)
In all images of $\mathbf{A B}$ and $\mathbf{A} \mathbf{B}$ systems, we observed many DNA structures in addition to the isolated 2-D crystals discussedabove. In many images the 2-D crystals appear to overlap, leading to discrete steps in thickness (Figure 11cef). The arrangement of crystals on the mica - solitary, overlapping, piled up like driftwood, ripped to shreds - depends sensitively upon DNA concentration and upon the sample preparation procedure, especially the wash step. Prominently, the background of every image contains small objects, which we assume to be associations of small numbers of DX units. Long, thin "rods" appear in some preparations (data not shown). These structures have not been characterized.

\subsection{Conclusions}

An exciting application of DNA self-assembly is to use periodic DNA lattices as scaffolds for bottom-up nanofabrication technologies, such as molecular electronic circuits (Robinson and Seeman 1987). However, for our discussion of molecular computation by self-assembly, our main concern is whether DNA self-assembly can be programmed to compute according to the logic of tilings discussed above.

The programmability of tile binding domains has already been demonstrated by Winfree et al. (1998a), where the four tiles of Figure 2 are implemented to produce DNA lattices with stripes of twice the periodicity. However, to achieve computational tilings such as the ones shown Figure 3, Figure 4, and Figure 5, we identified two additional requirements: that the input assemblies are formed first, and that rule tiles adsorb to the crystal only if they correctly match at least two binding domains. The former condition is easy to meet simply by mixing the input tiles first. Alternatively, the input tiles could be given longer sticky ends so that their interactions remain stable at a higher temperature; then, input tiles could be assembled at the higher temperature, and the rule tiles will only assemble once the temperature is lowered. The latter condition is more delicate, but should also be met by a proper balance of temperature and binding domain strength. The cooperativity of binding to adjacent binding domains has already been shown experimentally in a model system (Winfree et al. 1998b). Computer simulations suggest that error-free computational crystals will form in a large region of parameter space (Winfree preliminary, 1998).

Are other forms of self-assembly - in addition to 2D tiling appropriate for computing? Adleman made use of a one dimensional (1D) self-assembly process in his original work on molecular computation (Adleman 1994); there, DNA oligonucleotides with complementary $3^{\prime}$ and $5^{\prime}$ ends hybridized to form a combinatorial library of duplex DNA representing paths through a specified graph. Unfortunately, the 1D tiling problem is decidable, and thus 1D self-assembly is of limited computational power (Winfree et al. 1998b). Remarkably, although still limited, the 1D self-assembly of more complex units, such as double or triple crossover molecules (LaBean et al. in preparation, 1999), can perform considerably more sophisticated computations by making use of reporter strands that weave through 
each tile multiple times (Winfree et al. in preparation, 1999) Experimental work on these ideas is already in progress (LaBean et al. to appear). There have also been proposals for DNA based computing using the self-assembly of graph-like networks of branched DNA molecules (Jonoska et al. 1999, preliminary, 1998). These proposals are intriguing because they do not make use of rigid components; in fact, they rely upon the individual units being flexible. Finally, if 3D DNA crystals can be designed (Seeman 1982), it may be possible to self-assemble 3D tilings, where more efficient algorithms are possible.

\section{Acknowledgements}

This work would have been impossible without the sage advise of my thesis advisor, John Hopfield. John Abelson welcomed me into his laboratory, where this work was done. Ned Seeman taught me experimental science and gave me generous technical and conceptual advice on this project from its inception. Thanks to Anca Segall, Ely Rabani, and Bob Moision for instruction and advice on AFM imaging, and to the Beckman Institute Molecular Materials resource center for assistance and use of their AFM facilities. I also thank Paul Rothemund, Hui Wang, Len Adleman, John Reif, and many others whose discussions have been invaluable and inspirational. Computer models of the DX molecules used in this study, shown in Figure 6, were generated using NAMOT2 (Carter and Tung 96).

\section{References and Footnotes}

1. Adleman, L.M., Science 266, 1021-1024, 1994.

2. Bennett, C.H., Inter. J. of Theor. Pyhsics 21 (12), 905-940, 1982

3. Berger, R., Memoirs of the AMS 66, 1-72, 1966.

4. Binnig, G., Quate, C.F., and Gerber, Ch., Physical Review Letters 56 (9), 930 933, 1986.

5. Buchi, J.R., Mathematics Annalen 148, 201-213, 1962.

6. Carter, II, Eugene S., and Tung, Chang-Shung, CABIOS 12 (1), 25-30, 1996.

7. Fu, T.-J., and Seeman, N.C., Biochemistry 32, 3211-3220, 1993.

8. Grünbaum, B., and Shepard, G.C., Tilings and Patterns, W.H. Freeman and Company, New York, 1987

9. Hansma, H.G., Vesenka, J., Siegerist, C., Kelderman, G., Morrett, H., Sinsheimer, R.L., Elings, V., Bustamante, C., and Hansma, P.K., Science 256, 1180-1184, 1992.

10. Hjemfelt, A., and Ross, J., Physica D 84, 180-193, 1995.

11. Jonoska, N., Karl, S.A., and Saito, M., in Rubin and Wood (1999), 123-135.

12. Jonoska, N., Karl, S.A., and Saito, M., in Kari et al. (preliminary, 1998), 189200.

13. Kari, L., Rubin, H., and Wood, D.H., editors, Proceedings of the $4^{\text {th }}$ DIMACS Meeting on DNA Based Computers, held at the University of Pennsylvania, June 16-19, 1998, preliminary, 1998.

14. Kurtz, S.A., Mahaney, S.R., Royer, J.S., and Simon, J., Complexity Theory Retrospective II, 179-195, Hemaspaandra, L.A., and Selman, A.L., editors, Springer 1997.

15. LaBean, T.H., Winfree, E., and Reif, J.H., Proceedings of the $5^{\text {th }}$ DIMACS Meeting on DNA Based Computers, held at MIT, June 14-15, 1999, Providence RI, to appear.

16. LaBean, T.H., Yan, H., Kopatsch, J., Liu, F., Winfree, E., Reif, J.H., and Seeman, N.C., in preparation, 1999.

17. Landweber, L.F., and Baum, E.B., editors, DNA Based Computers II: DIMACS Workshop, June 10-12, 1996, Providence RI, 1998.

18. Lipton, R.J., Baum, E.B., editors, DNA Based Computers: DIMACS Workshop, April 4, 1995, Providence RI, 1996.

19. Magnasco, M.O., Physical Review Letters 78 (6), 1190-1193, 1997.

20. Robinson, B.H., and Seeman, N.C., Protein Engineering 1 (4), 295-300,
1987.

21. Robinson, R.M., Inventions Math., 12, 177-909, 1971.

22. Rubin, H., and Wood, D.H., editors, DNA Based Computers III: DIMACS Workshop, June 23-25, 1997.

23. Seeman, N.C., J. of Theor. Bio. 99 (2), 237-247, 1982.

24. Wang, H., Bell System Technical Journal 40, 1-42, 1961.

25. Wang, H., Proc. Symp. Math. Theory of Automata, 23-55, New York, 1963, Polytechnic Press.

26. Wang, H., Fundamenta Mathematicae 82, 295-305, 1975.

27. Weiss, R., Homsy, G.E., and Knight, T.F., Proceedings of the DIMACS Workshop on Evolution as Computation, held at Princeton, January 11-12, 1999, Landweber, L.F., and Winfree, E., editors. Springer-Verlag, to appear.

28. Winfree, E., in Lipton and Baum 199-221, 1996.

29. Winfree, E., in Kari et al. (preliminary, 1998).

30. Winfree, E., Eng, T., and Rozenberg, G., in preparation, 1999.

31. Winfree, E., Liu, F., Wenzler, L.A., and Seeman, N.C., Nature 394, 539-544, 1998a.

32. Winfree, E., Yang, X., Seeman, N.C., in Landweber and Baum, 1998b. 